\title{
A systematic review on the application of vascular endothelial growth factors in preeclampsia
}

\author{
Yan Liu ${ }^{1,2 \#}$, Meiying Ren ${ }^{1 \#}$, Xiaofang $\mathrm{Bi}^{3}$, Yuhua Fu${ }^{1}$, Xuefen Jing ${ }^{1}$, Hui Zhang ${ }^{1}$, Budao Cao ${ }^{1}$, \\ Cuifeng Wang ${ }^{1}$
}

${ }^{1}$ Department of Laboratory Medicine, the First Affiliated Hospital of Baotou Medical College, Inner Mongolia University of Science and Technology, Baotou, China; ${ }^{2}$ Graduate School of Baotou Medical College, Inner Mongolia University of Science and Technology, Baotou, China; ${ }^{3}$ Inner Mongolia Baogang Hospital, Baotou, China

Contributions: (I) Conception and design: Y Liu, C Wang, M Ren; (II) Administrative support: C Wang, M Ren; (III) Provision of study materials or patients: C Wang, Y Liu, M Ren, Y Fu; (IV) Collection and assembly of data: C Wang, Y Liu, X Bi, X Jing, H Zhang, B Cao; (V) Data analysis and interpretation: All authors; (VI) Manuscript writing: All authors; (VII) Final approval of manuscript: All authors.

\#These authors contributed equally to this work.

Correspondence to: Cuifeng Wang. Department of Laboratory Medicine, the First Affiliated Hospital of Baotou Medical College, Inner Mongolia University of Science and Technology, Baotou, China. Email: wangcuifeng1973@vip.sina.com.

Background: Hypertensive disorders of pregnancy (HDP) is a disease associated with elevated blood pressure during pregnancy, accounting for $5-10 \%$ of all pregnancies, which includes: gestational hypertension, preeclampsia (PE), eclampsia, chronic hypertension with superimposed $\mathrm{PE}$ and chronic hypertension. PE is the most prevalent type of HDP that seriously threatens the life and health of mothers and infants. In-depth exploration of the pathogenesis can play an early role in predicting the disease.

Methods: A literature search was conducted in PubMed, Embase, Google Scholar, and other databases in the article. It was investigated by searching for literature published between 1993 and March 2021; the subject terms included—-vascular endothelial growth factor", "preeclampsia", and "pathogenesis". In the article, the inclusion criteria of literature should meet the definition of PE. It was excluded as reviews, case reports, narrative reviews, and publications that lack key information.

Results: Vascular endothelial growth factor (VEGF) family factor research provides pivotal value for early clinical prediction of PE. Soluble fms-like tyrosine kinase-1 (sFlt-1)/placental growth factor (PlGF) became a marker for early prediction of PE. Through the included 51 articles, the analysis of VEGF in PE and its pathway factors was summarized to clarify the pathogenesis further and provide innovative ideas for future research directions and clinical diagnosis.

Discussion: A systematic review of the VEGF family in the pathogenesis of PE was concluded in the study to find angiogenesis markers of $\mathrm{PE}$ from the pathogenesis of the available literature. Therefore, early intervention of clinical diseases could reduce maternal complications and ensure the maximum health of mothers and babies. There are differences in the research results of factors in the VEGF family, and further research is needed to provide accurate clinical evidence.

Keywords: Preeclampsia (PE); vascular endothelial growth factor (VEGF); etiology

Submitted May 31, 2021. Accepted for publication Aug 17, 2021.

doi: 10.21037/apm-21-2109

View this article at: https://dx.doi.org/10.21037/apm-21-2109 


\section{Introduction}

In 1619, the word "eclampsia" appeared in Varandaeus' treatise on gynecology systematically described by Mauriceau first. Pregnant women with eclampsia would have spasm during pregnancy, and it is shown that the risk of eclampsia is higher for the multipara than the primipara in research (1). The term "preeclampsia" (PE) appeared in textbooks in 1903, and the classification criteria for different degrees of PE were gradually refined (2). Parturients with $\mathrm{PE}$ can cause fetal growth restriction and increase fetal mortality during the perinatal period. When the post-birth weight reduces $10 \%$ below, the mortality rate of the newborn will be increased (3). If the fetus is born and survives, the risk of coronary heart disease, stroke, metabolic syndrome, and other diseases will also be increased in life in the future (4). Currently, the effective treatment is childbirth. PE has familial heredity, and intervention measures have been taken to prevent $\mathrm{PE}$ and its complications (5). Therefore, early screening and intervention play a vital role in the prognosis of pregnant women and the growth of fetus.

In normal pregnancy, the villous cell trophoblast invades one-third of the myometrium, which reduces the endothelium and muscle fibers of the spiral artery. After the structure changes, the spiral artery's resistance, and sensitivity decrease, and it is not sensitive to vasoconstrictive substances; therefore, blood pressure will not increase (6). However, in pregnancy-induced hypertension, the process of invasion of the spiral arteries by trophoblast cells appears wrong. Current studies have shown (7): during pregnancy, shallower placental implantation could further lead to placental blood circulation disorder, release proinflammatory factors, and maternal inflammation causes endothelial cell damage and increases permeability, which will eventually increase maternal blood pressure. The absence of vascular endothelial growth factor (VEGF) in the podocytes blocks the gap in the diaphragm on the basement membrane to reduce the glomerular filtration rate, which could cause proteinuria. The current research shows (4): the development of hypertensive disorders in pregnancy is associated with the immune imbalance theory (manifested in the human leukocyte antigen area), the theory of oxidative stress, and the theory of genetics. These abnormal reactions are related to increased vascular permeability, leading to increased blood pressure and thrombosis, which further compensates for reducing blood flow in the subarteries caused by peripheral vasoconstriction (8). However, the pathogenesis of pregnancy-induced hypertension is still unclear, for which one factor cannot explain the etiology. In patients with $\mathrm{PE}$, thrombin induces increased mRNA and protein expression of protease-activated receptor- 1 on endothelial cells, predisposing to thrombus formation (9). Therefore, blood pressure during pregnancy increases, and endothelial cell damage may occur caused by complications. Endothelial cell-related factors include the family of VEGFs, which were including (10): VEGF-A, VEGF-B, VEGF-C, VEGF-D, VEGF-E, VEGF-F, placental growth factor (PlGF), and endocrine gland-derived VEGF (EGVEGF). The relationship between VEGF, its receptor, and $\mathrm{PE}$ was discussed in the article.

VEGF was the first independently isolated VEGF protein in 1989 by Ferrara and others. It is a $40-\mathrm{KD}$ heterodimeric glycoprotein formed by anti-parallel peptides, whose gene is located on chromosome $6 \mathrm{p} 21.3$ and belongs to the VEGF/platelet-derived growth factor gene family $(11,12)$. The VEGF ligand binds to the immunoglobulinlike domains 1-3 of its homologous VEGF receptor, which induces VEGF receptor activation through the symmetrical homotypic interaction between the membrane-proximal immunoglobulin-like domains 4-7 (13). VEGF-A of vascular endothelial cells is a high-affinity ligand of VEGF receptor 2 (VEGFR2, i.e., FLK/KDR), and the signal of VEGF-A is mediated by the activation of VEGFR2 (14). PlGF conveyed the signal transduction in cells through VEGF receptor 1 (VEGFR1, i.e., Flt-1). Meanwhile, the binding and activation of VEGF-A and VEGFR2 were increased (15). As the characteristics of specific binding to its receptor, VEGF initiates its pathway through one or two of the three endothelial receptor tyrosine kinases, which could play a vital role in forming blood vessels and lymphangion. However, there was weak tyrosine kinase activity of VEGFR1, which was functioned as the soluble fms-like tyrosine kinase-1 (sFlt-1) to produce an antagonist of vascular endothelium on the surface of endothelial cells (16). Because sFlt-1 lacked the transmembrane segments and intracellular structural domains, it could only bind to VEGF ligands. Free VEGF levels were downregulated to prevent VEGF from binding to its receptor, which could not bound to the receptor and PIGF further to cause placental ischemia in pregnant women, vascular endothelial damage and angiogenesis disorder that induces the elevation of blood pressure and proteinuria. Therefore, when the expression of VEGF is abnormal during pregnancy, the integrity of the blood vessel wall will be broken, along with the changed permeability, which 
will further trigger PE. A rat model study showed an imbalance of angiogenic factors in PE patients to reduce VEGF production. The anti-angiogenic factor sFlt-1 was reduced (17), consistent with the expression of factors in the above pathways. In addition, sFlt-1/PIGF in the pathway has a high negative predictive value. Research on sFlt-1/ PIGF intends to be an effective biomarker or drug target for the treatment and intervention of PE patients, which can provide new ideas for the early diagnosis and treatment of the disease.

We present the following article in accordance with the PRISMA reporting checklist (available at https://dx.doi. org/10.21037/apm-21-2109).

\section{Methods}

\section{Selection criteria}

In the article, the inclusion criteria of literature should meet the definition of PE: systolic blood pressure $\geq 140 \mathrm{mmHg}$ and/or diastolic blood pressure $\geq 90 \mathrm{mmHg}$ after 20 weeks of gestation with any of the following situations: proteinuria $\geq 0.3 \mathrm{~g} / 24 \mathrm{~h}$, or urinary protein/creatinine ratio $\geq 0.3$, or random urine protein $\geq+$; no proteinuria but accompanied by any organ or system involvement: abnormal changes in the heart, lung, liver, kidney and other vital organs, or blood system, digestive system, nerve system, placentafetal involvement, and others. The PE group was defined as the patients with mild $\mathrm{PE}$ in systolic blood pressure $140-<160 \mathrm{mmHg}$ and diastolic blood pressure $90-<110 \mathrm{mmHg}$ without serious complications, while severe complication group was defined as the patients with severe $\mathrm{PE}$ and complications in systolic blood pressure $\geq 160 \mathrm{mmHg}$ and diastolic blood pressure $\geq 110 \mathrm{mmHg}$. The involved topics were shown as etiology, pathogenesis, case-control studies, prospective research. It was excluded as reviews, case reports, narrative reviews, and publications that lack key information.

\section{Search method}

Searches were conducted in PubMed, Embase, and Google Scholar databases from January to March 2020, with the primary search terms as "Pre-Eclampsia", "Pregnancy Toxemia", "Edema-Proteinuria-Hypertension Gestosis" and "VEGF", "Vascular Endothelial Growth Factor-A", "VEGF-A", "Vascular Endothelial Growth Factor" and others. One thousand eight hundred and twenty-eight results were searched for the initial search results from 1993 to 2021. After further literature searches on clinical trials, randomized controls, and meta-analysis, 258 documents were included in the scope considered. Through the selection of inclusion and exclusion criteria, 51 articles were finally included.

\section{Data extraction}

The data extraction of this article includes the following content: title, first author and corresponding author, publication time, publication language, impact factor, the number of authors, detailed information of the case group population (age, systolic and diastolic blood pressure, BMI index, subtype classification of $\mathrm{PE}$ and severity of disease, presence or absence of previous disease history, clinical manifestations, intervention measures, risk prediction, influencing factors and others), research methods and research results.

\section{Results}

\section{VEGF and $P E$}

Existing studies have shown $(18,19)$ that compared with normal pregnant women, the reversible expression of VEGF gene mRNA in the placenta of PE pregnant women was increased, which could lead to high expression of VEGF factor, and the up-regulation of VEGF factor was considered the placental hypoxia response at the transcriptional level. Other researchers believe that (20): the expression level of $m i R-203$ in the placenta of PE patients was significantly increased $(\mathrm{P}<0.01)$, and the expression level of VEGFA was significantly decreased $(\mathrm{P}<0.01)$, which was negatively correlated $(r=0.2217 ; \mathrm{P}=0.0028)$ relationship in expression, indicating that the up-regulation of $m i R$ 203 may be related to the down-regulation of VEGFA. At present, there is still considerable controversy in the study of RNA expression in PE patients without the clarified pathogenesis. A large sample size and multi-population study should be further conducted to determine the exact role of RNA in PE patients. A Ahmed et al. believed the expression of VEGF in the peripheral blood of the PE group was downregulated compared with the normal pregnancy group and non-pregnant group $(\mathrm{P}<0.05)$. It was found that the mRNA expression level of VEGF in the placenta tissue of patients with $\mathrm{PE}$ was higher than the mRNA expression level in serum $(21,22)$. Senger first 
described it (23), and then through the developmental study of heterozygous VEGF-A knockout mice (VEGF-A+/-) (24), the mutant embryo dies at early embryogenesis on the 10th to 11th day, which proved VEGF-A has a powerful effect on promoting angiogenesis. Besides, it plays a critical role in cell proliferation, vasodilation, increasing vascular permeability, and inducing inflammatory cells to accumulate to the injury part (25).

\section{$V E G F-A$ gene polymorphism and PE}

Single nucleotide polymorphism (SNP) refers to a DNA sequence polymorphism caused by a single nucleotide variation (including the conversion or transversion, insertion, or deletion of a single base) at the genome level. SNP is widespread in the human genome, and changes in specific loci can cause disease. The polymorphism of the SNP locus in PE is manifested in VEGF-A, an essential angiogenesis stimulator. The two crucial SNP loci of VEGF-A are studied as below.

\section{Locus-634G/C and PE}

In the study of locus-643G/C and PE, Chen et al. had proved that maternal and fetal VEGF-A-634G/C locus polymorphism through a mixed study design combining case-parent design and case-control design (26): the dominant model of maternal-fetal VEGF-A-634G/C locus polymorphism $(G C+C C / G G)$ was significantly correlated with $\mathrm{PE}(\mathrm{OR}=1.85,95 \% \mathrm{CI}: 1.25-2.75$; OR $=1.90,95 \%$ CI: 1.28-2.83). In the locus, the expression of the $C C$ genotype was higher than the $C G+G G$ genotype. The risk of maternal $\mathrm{PE}$ could increase in the offspring with $C G$ or $C C$ genotype (OR $=1.84,95 \%$ CI: $1.18-2.86$; OR $=1.89,95 \%$ CI: $1.02-3.49)$. Therefore, the high genotype expression was related to increasing the risk of maternal PE. The results of gene-environment interaction analysis by log-linear model showed that passive smoking during pregnancy significantly affected the polymorphism of the $-634 G / C$ locus [likelihood ratio test $\left(\mathrm{P}_{\mathrm{LRT}}\right)=0.022$ ]. The maternal genotype was significantly correlated with $\mathrm{PE}$ risk $(\mathrm{P}=0.028) .95 \% \mathrm{CI}:(0.40-3.18 ; 0.11-1.10)$ of $G C$ and $C C$ genotypes shows this factor is not associated with $\mathrm{PE}$; whether there is an association between their geneenvironment interactions should be further investigated (25-27). In the RMDR interaction model study (28), the -1562CT genotype with VEGF-634CC, -634GG, and matrix metalloproteinase-9 genes are more common in the $\mathrm{PE}$ group than in the normal pregnancy group. The complex interactions between genes and the environment, genes and genes are more important than the independent effects of any susceptible gene. The above studies have investigated the interaction of $-634 G / C$ locus from a maternal-infant perspective and gene-environment interactions with variable results. Many factors should be considered and a large sample study should be carried out.

\section{Locus $+936 \mathrm{C} / \mathrm{T}$ and $\mathrm{PE}$}

Locus $+936 \mathrm{C} / \mathrm{T}$ is a $3^{\prime}$ untranslated region locus of the VEGF gene. A Korean study showed that (29): locus $+936 C / T$ was significantly different in case-control studies $(\mathrm{P}<0.001)$, and the frequency of patients with the gene was significantly higher than the control group $(\mathrm{OR}=2.06$; 95\% CI: 1.38-3.08), which has shown the locus was a risk factor for PE. A study in the Philippines (30): the alleles of VEGF-A and locus $+936 \mathrm{C} / T$ were further explored in locus studies. Among them, the $\mathrm{C}$ allele was a risk factor for $\mathrm{PE}(\mathrm{OR}=1.648,95 \% \mathrm{CI}: 1.03-2.62)$, and the $\mathrm{T}$ allele was its protective factor ( $\mathrm{OR}=0.61,95 \% \mathrm{CI}$ : $0.389-0.975$ ). In another study (31), the risk of severe PE was increased when the pregnant woman was a homozygous $T T$ genotype carrier $(\mathrm{P}=0.03)$. When locus $+936 \mathrm{C} / T$ allele was present in the mother and newborn, then the risk of pregnancyinduced hypertension could significantly increase. There are still significant differences in the study of locus $+936 \mathrm{C} /$ $T$ in PE. Whether it is associated with the geographical environment remains unclear, and further multi-regional and multi-factor research should be conducted.

\section{Other angiogenic factors and $P E$}

\section{PIGF and PE}

PlGF is a pro-angiogenic factor of the VEGF family, located on chromosome 14q14, and encoded four PlGF subtypes expressed in the placenta. PlGF-1 and PlGF-2 are expressed during pregnancy (32). PlGF increases VEGF activity by competitively binding to the VEGF-1 receptor so that VEGF binds to VEGF-2 with stronger tyrosine kinase activity (33). Although the mice with the knockout PlGF gene were performing normally in the experiment, some studies have shown that there are nuanced differences in the development of cerebral blood vessels in the offspring of patients with $\mathrm{PE}$, which can show that PlGF has an important influence on blood vessel development (34). In women who develop PE,the concentration of PIGF 
decreases in early pregnancy,and its level is negatively correlated with the sverity of the disease. PIGF levels have a predictive effect on early-onset PE (35). PIGF is overexpression during 15 to 20 weeks of normal gestation. The expression is lower than the normal pregnant women during 15-20 weeks of PE period. In a prospective study, the level of PlGF during normal pregnancy was $1.17 \pm 0.67$, and the level of the PE group was $0.65 \pm 0.30$. There was a significant difference between the two groups $(\mathrm{P}=0.014)$ (36). The decrease of PlGF in pregnant women with early onset $\mathrm{PE}$ may damage the infiltration of trophoblastic cells, reduce placental angiogenesis to cause placental ischemia and hypoxia, with a significant increase of sFlt- 1 . The elevated sFlt-1 levels will also affect PlGF-producing activity $(37,38)$. PlGF decreases in the first 3 months of pregnancy, and the decrease in expression level precedes its clinical presentation. However, PE can be predicted based on PlGF levels alone, with a false positive rate of $5 \%$ and a sensitivity of $32 \%$ (39). If the further combination of medical history, ultrasound examination, and the ratio of sFlt-1:PlGF can reduce the false-positive rate. However, the method has not been widely used in clinical practice. In another study (40), showed that low concentrations of PlGF $(<100 \mathrm{pg} / \mathrm{mL})$ have higher accuracy in identifying $\mathrm{PE}$ requiring delivery within 14 days. Among them, delivery before 35 weeks of gestation has high sensitivity (94.9\%) and negative predictive value (98.3\%). In women with suspected PE, the measurement of PlGF can shorten the diagnosis time of PE by about 2 days, which could provide a reliable basis for uncertain diagnosis. As a predictive indicator, PlGF led to a reduction in adverse maternal outcomes, for which prenatal care could be significantly improved, and serious complications could be avoided.

\section{Flt-1 and PE}

The alteration of genes in the fetal genome near the Flt region can cause PE. It was found that the locus rs12050029 was an independent risk factor related to PE. A recent large-scale genome-wide analysis showed that (41): the SNP at Flt-1 locus rs 4769613 on Chromosome 13 of the fetal genome was significantly associated with $\mathrm{PE}$ $\left(\mathrm{P}=3.6 \times 10^{-4}\right)$, the patient with late-onset $\mathrm{PE}$ and birth weight less than $10 \%$ in pregnancies could be significantly impacted by $\mathrm{C}$ allele of $r s 4769613$ (OR $=1.26, \mathrm{P}=1.2 \times 10^{-7}$ in the case-control). Both rs4769613 and rs12050029 are located in the placental enhancer region near Flt-1, and to some extent affect the transcription of Flt-1 through changes in gene transcription (42). The study of the Flt-1 locus could help clarify the source of sFlt-1 further. sFlt-1 is a spliced variant of VEGFR1 (Flt-1). In PE, placental perfusion is reduced by the invasion of the abnormal cytotrophoblast of spiral arteries. Alternative splicing of pre-mRNA encoding Flt-1 results in overrelease of sFlt-1 in the maternal circulation. Because sFlt1 contains the extracellular ligand domain of VEGFR1 (43), sFlt-1 antagonizes VEGF and PlGF. The binding of the two to their receptors can be inhibited to further the effect of angiogenesis signal pathways (44), resulting in endothelial function and systemic vascular dysfunction. Meanwhile, experiments have shown (45) that genetic polymorphisms in the fetal genome may increase the risk of disease for the mother. In 2003, some scholars found that the level of sFlt1 in the serum of PE patients increased. When sFlt-1 was administered to pregnant rats, the symptoms of $\mathrm{PE}$ would occur. Later, another experiment proved: the level of sFlt1 is related to the severity of $\mathrm{PE}$, and its concentration decreases after the termination of pregnancy. sFlt- 1 is a marker of placental insufficiency, which may be related to maternal and infant disease risk (46).

\section{sFlt-1/PIGF and PE}

Recently, sFlt-1/PlGF has been included in the official guidelines for predicting PE. However, the role of the ratio in prediction and diagnosis has only been verified in the European population. The published literature (47): the circulating sFlt-1/PlGF $<38$ has a $99.3 \%$ negative predictive value for the onset of $\mathrm{PE}$ within 1 week. It had clinical value in predicting the absence of adverse maternal or fetal outcomes in the short term. Compared with normal pregnancy (48), the fetal growth is restricted in PE patients, with a significantly higher ratio of plasma sFlt-1/PlGF. The cut-off value of the sFlt-1/PlGF ratio of 35 , the sensitivity of $89 \%$, and the specificity of $95 \%$ are defined in the case group. Compared with sFlt-1/ PlGF in plasma, the sensitivity of $71 \%$ and specificity of $86 \%$ of urine sFlt-1/PlGF ratio decreased. In the first study of sFlt-1/PlGF through different ethnic populations in Xinjiang, China (49), the ratio of sFlt-1/PlGF in PE group, severe $\mathrm{PE}$ group, and control group in non-Han Chinese $(21.76,27.53)$ were higher than those of Han Chinese $(6.06,13.85)$, among which the ratio of sFlt-1/ PlGF of Uygur nationality was higher than that of other nationalities. Meanwhile, it was found that the best sFlt1/PlGF cut-off value for distinguishing $\mathrm{PE}$ was defined as $>38.83$, the area under curve (AUC) value of receiver operator characteristic (ROC) curve was $0.802(57.4 \%$ of 
sensitivity, $95.1 \%$ of specificity), and the sFlt-1/PlGF ratio was relatively higher than the authenticity of the single detection, and sFlt-1/PlGF can significantly improve the prediction accuracy of $\mathrm{PE}$ risk (50). The sensitivity and specificity of sFlt-1/PlGF in Xinjiang were not higher than in other countries, which may be related to the small sample size and the regional limitations. The latest guidelines of the International Association for the Study of hypertensive disorders of pregnancy (HDP) recommend that large-scale prospective studies should be further conducted before the large-scale application of angiogenesis marker assays to clinical use (51).

\section{Discussion}

In summary, the effective screening and monitoring of pregnant women at risk of $\mathrm{PE}$ is necessary. $\mathrm{PE}$ will be associated with complications including urinary protein, elevated blood pressure, headache, and edema after 20 weeks of gestation. However, these symptoms cannot be used for clinical judgment in early pregnancy. The intervention treatment should be conducted as early as possible to protect the health of mothers and babies to the greatest extent, based on the research on the pathogenesis of $\mathrm{PE}$, which could reduce maternal complications. By studying genetic polymorphisms of critical factors in the VEGF family that affect vascular growth and development, an association with $\mathrm{PE}$ has been found, while there are still differences in research results. PlGF, sFlt-1, and the ratio of two indexes have become the hot topics of research in recent years. Their application in the clinic as angiogenic markers for PE has become possible. Further research should be conducted in the early stages of research to provide clinical diagnosis and treatment value. The abnormal expression of VEGF family related proteins and mRNA have important clinical value for the early diagnosis of PE. sFlt-1/PIGF can be used as an important indicator for early diagnosis of $\mathrm{PE}$, with high clinical value. sFlt-1/PIGF will be the central research topic in the future of $\mathrm{PE}$.

\section{Acknowledgments}

Funding: National Development and Reform Commission Enhancement of Manufacturing Core Competitiveness Project [Grant number: Development and Reform Industry (2017) No. 2000/Development and Reform Industry (2019) No. 158].

\section{Footnote}

Reporting Checklist: The authors have completed the PRISMA reporting checklist. Available at https://dx.doi. org/10.21037/apm-21-2109

Conflicts of Interest: All authors have completed the ICMJE uniform disclosure form (available at https:// dx.doi.org/10.21037/apm-21-2109). CW reports funding from National Development and Reform Commission Enhancement of Manufacturing Core Competitiveness Project [Grant number: Development and Reform Industry (2017) No. 2000/Development and Reform Industry (2019) No. 158]. The other authors have no conflicts of interest to declare.

Ethical Statement: The authors are accountable for all aspects of the work in ensuring that questions related to the accuracy or integrity of any part of the work are appropriately investigated and resolved.

Open Access Statement: This is an Open Access article distributed in accordance with the Creative Commons Attribution-NonCommercial-NoDerivs 4.0 International License (CC BY-NC-ND 4.0), which permits the noncommercial replication and distribution of the article with the strict proviso that no changes or edits are made and the original work is properly cited (including links to both the formal publication through the relevant DOI and the license). See: https://creativecommons.org/licenses/by-nc-nd/4.0/.

\section{References}

1. Bell MJ. A historical overview of preeclampsia-eclampsia. J Obstet Gynecol Neonatal Nurs 2010;39:510-8.

2. Sones JL, Davisson RL. Preeclampsia, of mice and women. Physiol Genomics 2016;48:565-72.

3. Backes CH, Markham K, Moorehead P, et al. Maternal preeclampsia and neonatal outcomes. J Pregnancy 2011;2011:214365.

4. Uzan J, Carbonnel M, Piconne O, et al. Pre-eclampsia: pathophysiology, diagnosis, and management. Vasc Health Risk Manag 2011;7:467-74.

5. Wright D, Rolnik DL, Syngelaki A, et al. Aspirin for Evidence-Based Preeclampsia Prevention trial: effect of aspirin on length of stay in the neonatal intensive care unit. Am J Obstet Gynecol 2018;218:612.e1-6. 
6. Rana S, Lemoine E, Granger JP, et al. Preeclampsia: pathophysiology, challenges, and perspectives. Circ Res 2019;124:1094-112.

7. Borzychowski AM, Sargent IL, Redman CW. Inflammation and pre-eclampsia. Semin Fetal Neonatal Med 2006;11:309-16.

8. Roberts JM. Endothelial dysfunction in preeclampsia. Semin Reprod Endocrinol 1998;16:5-15.

9. Ellis CA, Malik AB, Gilchrist A, et al. Thrombin induces proteinase-activated receptor-1 gene expression in endothelial cells via activation of Gi-linked Ras/ mitogen-activated protein kinase pathway. J Biol Chem 1999;274:13718-27.

10. Samson M, Peale FV Jr, Frantz G, et al. Human endocrine gland-derived vascular endothelial growth factor: expression early in development and in Leydig cell tumors suggests roles in normal and pathological testis angiogenesis. J Clin Endocrinol Metab 2004;89:4078-88.

11. Yamazaki Y, Morita T. Molecular and functional diversity of vascular endothelial growth factors. Mol Divers 2006;10:515-27.

12. Takahashi $H$, Shibuya $M$. The vascular endothelial growth factor (VEGF)/VEGF receptor system and its role under physiological and pathological conditions. Clin Sci (Lond) 2005;109:227-41.

13. Markovic-Mueller S, Stuttfeld E, Asthana M, et al. Structure of the full-length VEGFR-1 extracellular domain in complex with VEGF-A. Structure 2017;25:341-52.

14. Simons M, Gordon E, Claesson-Welsh L. Mechanisms and regulation of endothelial VEGF receptor signalling. Nat Rev Mol Cell Biol 2016;17:611-25.

15. Robciuc MR, Kivelä R, Williams IM, et al. VEGFB/ VEGFR1-induced expansion of adipose vasculature counteracts obesity and related metabolic complications. Cell Metab 2016;23:712-24.

16. Shibuya M, Claesson-Welsh L. Signal transduction by VEGF receptors in regulation of angiogenesis and lymphangiogenesis. Exp Cell Res 2006;312:549-60.

17. Gilbert JS, Gilbert SA, Arany M, et al. Hypertension produced by placental ischemia in pregnant rats is associated with increased soluble endoglin expression. Hypertension 2009;53:399-403.

18. Robinson CJ, Stringer SE. The splice variants of vascular endothelial growth factor (VEGF) and their receptors. J Cell Sci 2001;114:853-65.

19. Garza-Veloz I, Castruita-De la Rosa C, Cortes-Flores R, et al. No association between polymorphisms/haplotypes of the vascular endothelial growth factor gene and preeclampsia. BMC Pregnancy Childbirth 2011;11:35.

20. Liu F, $\mathrm{Wu} \mathrm{K}, \mathrm{Wu} \mathrm{W}$, et al. miR-203 contributes to pre-eclampsia via inhibition of VEGFA expression. Mol Med Rep 2018;17:5627-34.

21. A Ahmed M, I Alqosaibi A, Mohamed MA, et al. Evaluation of some cytokines and gene expressions in preeclampsia. Pak J Biol Sci 2019;22:148-53.

22. Tandon V, Hiwale S, Amle D, et al. Assessment of serum vascular endothelial growth factor levels in pregnancy-induced hypertension patients. J Pregnancy 2017;2017:3179670.

23. Hoeben A, Landuyt B, Highley MS, et al. Vascular endothelial growth factor and angiogenesis. Pharmacol Rev 2004;56:549-80.

24. Shibuya M. Vascular endothelial growth factor (VEGF) and its receptor (VEGFR) signaling in angiogenesis: a crucial target for anti- and pro-angiogenic therapies. Genes Cancer 2011;2:1097-105.

25. Koch S, Claesson-Welsh L. Signal transduction by vascular endothelial growth factor receptors. Cold Spring Harb Perspect Med 2012;2:a006502.

26. Chen XZ, Yu SJ, Wei MH, et al. Effects of maternal and fetal vascular endothelial growth factor a single nucleotide polymorphisms on pre-eclampsia: a hybrid design study. Cytokine 2020;127:154995.

27. Keshavarzi F, Shahrakipoor M, Teimoori B, et al. Association of the placental VEGF promoter polymorphisms and VEGF mRNA expression with preeclampsia. Clin Exp Hypertens 2019;41:274-9.

28. Luizon MR, Sandrim VC, Palei AC, et al. Epistasis among eNOS, MMP-9 and VEGF maternal genotypes in hypertensive disorders of pregnancy. Hypertens Res 2012;35:917-21.

29. Shim JY, Jun JK, Jung BK, et al. Vascular endothelial growth factor gene $+936 \mathrm{C} / \mathrm{T}$ polymorphism is associated with preeclampsia in Korean women. Am J Obstet Gynecol 2007;197:271.e1-4.

30. Amosco MD, Villar VA, Naniong JM, et al. VEGF-A and VEGFR1 SNPs associate with preeclampsia in a Philippine population. Clin Exp Hypertens 2016;38:578-85.

31. Procopciuc LM, Caracostea G, Zaharie G, et al. Maternal/ newborn VEGF-C936T interaction and its influence on the risk, severity and prognosis of preeclampsia, as well as on the maternal angiogenic profile. J Matern Fetal Neonatal Med 2014;27:1754-60.

32. Arad A, Nammouz S, Nov Y, et al. The expression of neuropilin-1 in human placentas from normal 
and preeclamptic pregnancies. Int J Gynecol Pathol 2017;36:42-9.

33. Gobble RM, Groesch KA, Chang M, et al. Differential regulation of human PIGF gene expression in trophoblast and nontrophoblast cells by oxygen tension. Placenta 2009;30:869-75.

34. Dang F, Croy BA, Stroman PW, et al. Impacts of preeclampsia on the brain of the offspring. Rev Bras Ginecol Obstet 2016;38:416-22.

35. Di Lorenzo G, Ceccarello $M$, Cecotti V, et al. First trimester maternal serum PIGF, free -hCG, PAPP-A, PP13 , uterine artery Doppler and maternal history for the prediction of preeclampsia. Placenta 2012;33:495-501.

36. Sultana MT, Begum F, Akhter N, et al. Comparison of placental growth factor levels in maternal serum at early pregnancy between normotensive pregnant women and patients with hypertensive disorders in pregnancy. Mymensingh Med J 2020;29:609-15.

37. Lecarpentier E, Tsatsaris V. Angiogenic balance (sFlt1/PlGF) and preeclampsia. Ann Endocrinol (Paris) 2016;77:97-100.

38. Song C, Xie S, Wang J, et al. Association of angiotensinogen gene polymorphisms and angiogenic factors with preeclampsia in Chinese women. Gynecol Obstet Invest 2013;76:64-8.

39. Kleinrouweler CE, Wiegerinck MM, Ris-Stalpers C, et al. Accuracy of circulating placental growth factor, vascular endothelial growth factor, soluble fms-like tyrosine kinase 1 and soluble endoglin in the prediction of pre-eclampsia: a systematic review and meta-analysis. BJOG 2012;119:778-87.

40. Duhig KE, Myers J, Seed PT, et al. Placental growth factor testing to assess women with suspected pre-eclampsia: a multicentre, pragmatic, stepped-wedge cluster-randomised controlled trial. Lancet 2019;393:1807-18.

41. McGinnis R, Steinthorsdottir V, Williams NO, et al. Variants in the fetal genome near FLT1 are associated with risk of preeclampsia. Nat Genet 2017;49:1255-60.

42. Gray KJ, Saxena R, Karumanchi SA. Genetic

Cite this article as: Liu Y, Ren M, Bi X, Fu Y, Jing X, Zhang H, Cao B, Wang C. A systematic review on the application of vascular endothelial growth factors in preeclampsia. Ann Palliat Med 2021;10(8):9259-9266. doi: 10.21037/apm-21-2109 predisposition to preeclampsia is conferred by fetal DNA variants near FLT1, a gene involved in the regulation of angiogenesis. Am J Obstet Gynecol 2018;218:211-8.

43. Kendall RL, Thomas KA. Inhibition of vascular endothelial cell growth factor activity by an endogenously encoded soluble receptor. Proc Natl Acad Sci U S A 1993;90:10705-9.

44. Clark DE, Smith SK, He Y, et al. A vascular endothelial growth factor antagonist is produced by the human placenta and released into the maternal circulation. Biol Reprod 1998;59:1540-8.

45. Maynard SE, Min JY, Merchan J, et al. Excess placental soluble fms-like tyrosine kinase 1 (sFlt1) may contribute to endothelial dysfunction, hypertension, and proteinuria in preeclampsia. J Clin Invest 2003;111:649-58.

46. Levine RJ, Maynard SE, Qian C, et al. Circulating angiogenic factors and the risk of preeclampsia. N Engl J Med 2004;350:672-83.

47. Zeisler H, Llurba E, Chantraine F, et al. Predictive value of the sFlt-1:PlGF ratio in women with suspected preeclampsia. N Engl J Med 2016;374:13-22.

48. Zhang K, Zen M, Popovic NL, et al. Urinary placental growth factor in preeclampsia and fetal growth restriction: an alternative to circulating biomarkers? J Obstet Gynaecol Res 2019;45:1828-36.

49. Ding G, Liping L, Moli D, et al. A study of the association between the sFlt-1/PIGF ratio and preeclampsia in Xinjiang Uygur Autonomous Region of China. Artif Cells Nanomed Biotechnol 2018;46:S281-6.

50. Perry H, Binder J, Kalafat E, et al. Angiogenic marker prognostic models in pregnant women with hypertension. Hypertension 2020;75:755-61.

51. Brown MA, Magee LA, Kenny LC, et al. Hypertensive disorders of pregnancy: ISSHP classification, diagnosis, and management recommendations for international practice. Hypertension 2018;72:24-43.

(English Language Editor: J. Chapnick) 